\title{
Dating Alphaproteobacteria evolution with eukaryotic fossils
}

2

\author{
Sishuo Wang ${ }^{1}$, Haiwei Luo ${ }^{1 *}$
}

3

$4 \quad{ }^{1}$ Simon F. S. Li Marine Science Laboratory, School of Life Sciences and State Key

5 Laboratory of Agrobiotechnology, The Chinese University of Hong Kong, Shatin,

6 Hong Kong SAR

7

\section{$8 *$ Corresponding author:}

9 Haiwei Luo

10 The Chinese University of Hong Kong

11 Shatin, Hong Kong SAR

12 Phone: (+852) 39436121

13 E-mail: hluo2006@gmail.com

14

15 Keywords: Alphaproteobacteria, Rickettsiales, molecular dating, microbial evolution,

16 host-microbe coevolution, Holosporales

17 


\section{Abstract}

19 Elucidating the timescale of evolution of bacteria is key to testing hypotheses on their

20 co-evolution with eukaryotic hosts, which, however, is largely limited by the scarcity

21 of bacterial fossils. Here, we incorporate eukaryotic fossils to date the divergence

22 times of Alphaproteobacteria, based on the endosymbiosis theory that mitochondria

23 evolved from an alphaproteobacterial lineage. We estimate that Alphaproteobacteria

24 arose $~ 1900$ million years (Ma) ago, followed by rapid divergence of their major

25 clades. We show that the origin of Rickettsiales, an order of obligate intracellular

26 bacteria whose hosts are mostly animals, predates the emergence of animals for $\sim 700$

27 Ma but coincides with that of eukaryotes. This, together with reconstruction of

28 ancestral hosts, strongly suggests that early Rickettsiales lineages had established

29 previously underappreciated interactions with unicellular eukaryotes. Our

30 mitochondria-based approach displays higher precision and robustness to

31 uncertainties compared with the traditional strategy using cyanobacterial fossils, and

32 suggests that previous applications using divergence times of the modern hosts of

33 symbiotic bacteria to date the bacterial tree of life may need to be revisited. 


\section{Main}

36 The Alphaproteobacteria is one of the largest grsoups within bacteria (Ettema and

37 Andersson, 2009) and of great evolutionary significance for holding the origin of the

38 mitochondrion (Wang and Wu, 2015; Yang et al., 1985). The Alphaproteobacteria has

39 extensively diversified since its ancient origin, and comprised some of the most

40 environmentally abundant and metabolically diverse organisms on Earth (Boussau et

41 al., 2004; Ettema and Andersson, 2009; Luo et al., 2013). The intimate association

42 between some alphaproteobacterial lineages and eukaryotes is of central importance

43 for agricultural (e.g., rhizobia) and medical (e.g., rickettsia) applications. This makes

44 Alphaproteobacteria a promising system to study the timing of bacterial evolution and

45 their correlation with geological, ecological and evolutionary events (Luo et al., 2013;

46 Sun et al., 2017; Wang et al., 2020). However, the traditional way of divergence time

47 estimation has several limits when applied to Alphaproteobacteria and other

48 prokaryotes. First, fossil records of bacteria are extremely scarce and controversial in

49 terms of their estimated dates (Schirrmeister et al., 2016). Second, the most widely

50 used prokaryotic fossils are from cyanobacteria, but the long evolutionary distance

51 between cyanobacteria and other bacteria causes large uncertainties in dating (Nei et

52 al., 2001). Last, some studies assumed a strict relationship of bacteria-host evolution,

53 and calibrated the evolution of pathogenic/symbiotic bacteria based on the divergence 
54 time of their modern hosts (mostly animals and plants). However, this precludes the

55 possibility of host switching, which could occur frequently during evolutionary

56 processes spanning millions of years (Wang et al., 2020). Due to these challenges, the

57 origin time of Alphaproteobacteria estimated by previous studies varies from less

58 than 600 million years (Ma) to more than $2000 \mathrm{Ma}$ (Battistuzzi et al., 2004;

59 Battistuzzi and Hedges, 2009; Chriki-Adeeb and Chriki, 2016; Luo et al., 2013),

60 making any narratives based on its evolutionary timing contentious.

61 Recently, horizontal gene transfer (HGT) has been suggested to have great

62 potential in dating the evolution of bacteria (Davín et al., 2018; Wolfe and Fournier,

63 2018). Briefly, if the donor of an HGT event does not have fossil records while the

64 recipient does, the temporal information recorded in the recipient can be transferred to

65 date the evolution of the donor group (and vice versa), thereby bypassing the paucity

66 of fossils in the donor lineage. Inspired by this idea, we developed a new strategy to

67 date the divergence times of Alphaproteobacteria based on the endosymbiosis theory

68 that the mitochondrion was transferred to a host nucleus from a bacterial lineage

69 (Sagan, 1967), which was later shown to be within (Fan et al., 2020; Wang and Wu,

70 2015; Yang et al., 1985) or closely related to (Martijn et al., 2018)

71 Alphaproteobacteria by modern phylogenetic analysis. As mitochondria are

72 characteristic of eukaryotes, we took advantages of eukaryotic fossils to anchor the 
73 divergence time of Alphaproteobacteria in a tree integrating both alphaproteobacterial

74 and mitochondrial lineages.

75 We first reconstructed a phylogenomic tree of 80 carefully selected

76 Alphaproteobacteria and mitochondrial genomes using 24 conserved genes based on

77 prior phylogenomics studies (Martijn et al., 2018; Muñoz-Gómez et al., 2019) (see

78 Methods and Supplementary Note S1.1). We employed rigorous approaches to

79 delineate phylogenetic artefacts caused by long branch attraction and compositional

80 heterogeneity (see Methods), and obtained results consistent with recent studies where

81 i) Rickettsiales, Holosporales and Pelagibacterales (SAR11) had independent origins

82 (Muñoz-Gómez et al., 2019), and ii) mitochondria branched as a sister to

83 Alphaproteobacteria (Martijn et al., 2018) (Fig. S1A). We also tested the impact of

84 alternative topologies on dating (Fig. S1C; see below). We compiled two datasets to

85 estimate the time divergences within the Alphaproteobacteria calibrated by eukaryotic

86 fossils with relaxed molecular clock (Drummond et al., 2006), which accounts for

87 substitution rate variations among branches. The first dataset, which we referred to as

88 the mito-encoded dataset, was based on the aforementioned 24 conserved gene

89 encoded by mitochondrial genomes (Dataset S1), and the mitochondrial lineages

90 mainly comprised species of green plants, red algae and jakobids, whose

91 mitochondrial genomes are both gene-rich and relatively slowly-evolving (Martijn et 
92 al., 2018; Rodríguez-Ezpeleta and Embley, 2012). Four high-confidence fossils from

93 land plants and red algae were used as the calibration points (Supplementary Note

94 S2.1; Fig. S2A). The second dataset, referred to as the nuclear-encoded dataset, was

95 based on 22 mitochondria-derived genes that had been transferred to the nuclear

96 genome identified by Wang and Wu, 2015 (Dataset S1; see Methods). This dataset not

97 only circumvented the problem that in many eukaryotes genes encoded by

98 mitochondrial genomes are few (e.g., apicomplexans and dinoflagellates) or

99 fast-evolving (e.g., animals and fungi) (Roger et al., 2017), but integrated six

100 additional eukaryotic fossils (Fig. S2A; Supplementary Note S2.1), allowing directly

101 comparing the divergence time between symbiotic bacteria and their eukaryotic hosts

102 on the same tree.

103 We selected a best-practiced scheme based on systematic comparisons of

104 different combinations of parameters of MCMCTree for the mito- and

105 nuclear-encoded datasets (see Methods and Supplementary Note S1.2). Similar

106 divergence times were recovered for most nodes between the two datasets, although

107 the mito-encoded dataset estimated older ages for deep nodes (Fig. 1). As shown in

108 the infinite-sites plots (up- and bottom-left panels in Fig. 1), the posterior mean ages

109 versus 95\% HPD (highest posterior density) widths approached a straight line,

110 suggesting that the uncertainty in time estimate was predominantly caused by the 
111 uncertainty associated with fossil calibrations (Rannala and Yang, 2007). The

112 estimated ages in the nuclear-encoded dataset exhibited smaller 95\% HPD intervals,

113 hence smaller uncertainties, likely due to their more calibration information compared

114 with the mito-encoded dataset.

115 Most alphaproteobacterial orders diverged 1500-1000 Mya, and Rickettsiales and

116 Pelagibacterales appeared to be the oldest and youngest alphaproteobacterial orders,

117 respectively (Fig. 1). The origin times of Rickettsiales, an obligate endosymbiont

118 lineage whose hosts cover diverse eukaryotes but mostly animals (Merhej and Raoult,

119 2011), were estimated to be $1771 \mathrm{Ma}$ (95\% HPD 1972-1565 Ma) and $1523 \mathrm{Ma}(95 \%$

120 HPD 1677-1368 Ma) using the mito- and nuclear-encoded datasets, respectively (Fig.

121 1). A merit of our mitochondria-based method is that divergence times of the

122 eukaryotic hosts and of the host-associated bacteria can be simultaneously estimated.

123 As shown in Fig. 1, we dated the origin of animals to be 803 Ma (95\% HPD 844-752

124 Ma), consistent with previous dating analyses (Betts et al., 2018; Dos Reis et al., 2015;

125 Parfrey et al., 2011), but not others (Hedges et al., 2004). We also estimated that

126 mitochondrial lineages diverged from Alphaproteobacteria $1900 \mathrm{Ma}$ ago and that the

127 last common ancestor (LCA) of mitochondria occurred 1550 Ma ago (Fig. 1). Thus,

128 the origin of Rickettsiales likely predated the evolutionary emergence of animals for

$129 \sim 700$ Ma but coincided with the mitochondrial endosymbiosis process and the 
130

131

132

133

134

135

136

137

138

139

140

141

142

143

144

145

146

147

occurrence of the LCA of eukaryotes, according to our (Fig. 1) and others' estimates

(Betts et al., 2018; Eme et al., 2014; Parfrey et al., 2011), and fossil records (reviewed

in Butterfield, 2015). This agrees with recent findings of an increasingly broad range

of protistan hosts of Rickettsiales (reviewed in Castelli et al., 2016), and suggests that

host switches to animals from protists occurred later in the evolution of Rickettsiales.

The origin time of Holosporales, another important endosymbiotic lineage in

Alphaproteobacteria whose extant members are mostly endonuclear parasites of the

ciliate Paramecium (Schrallhammer et al., 2018), dated back to 1379 Ma (95\% HPD

1559-1201 Ma) or $1212 \mathrm{Ma}$ (95\% HPD 1353-1067 Ma), respectively, based on the

mito- or nuclear-encoded datasets. This implies that the origin of Holosporales

roughly coincided with the that of ciliates, which dated back to $\sim 1150 \mathrm{Ma}$ according

to other estimates (Fernandes and Schrago, 2019; Parfrey et al., 2011). While the

above analyses were based on amino acid sequences using MCMCTree, the basic

patterns held similar with PhyloBayes or using coding sequences (Fig. S3).

We assessed the impact of uncertainties in Bayesian relaxed molecular clock

time estimation, including the disparity between fossil evidence and molecular clock

estimates, root age, across-branch rate variation, sequence partitioning, and clock

model, on the posterior ages (Dataset S2). When we used only Phanerozoic fossils

and excluded all Proterozoic fossils (which are thought controversial by some), the 
149 estimated ages of most nodes were shifted towards the present for the mito-encoded

150 dataset but not for the nuclear-encoded dataset (Phan in Figs. 2A, S4). Removing

151 potentially controversial maximum age constraints led to minor changes in the

152 posterior ages (Max-1 in Figs. 2A, S4). Using more conservative calibrations of the

153 root pushed the time estimates to be slightly older for the mito-encoded dataset

154 (Root-1 in Figs. 2A, S4). Allowing larger rate variation among branches resulted in

155 highly consistent results with the best-practiced scheme (Sigma in Fig. S5).

156 Decreasing the number of partitions resulted in decreased precision, as indicated by

157 the increase of the slope in infinite-sites plots (Fig. S6), but the estimated dates

158 remained similar (Single partition in Fig. 2A). The largest changes in the posterior

159 ages were obtained when the independent rates (IR) instead of autocorrelated rates

160 (AR) clock model was used: the divergence times of most alphaproteobacterial orders

161 were shifted towards the present by $\sim 20 \%$ (IR in Figs. 2A, S4). Collectively, the

162 composite of the ages estimated from six different analyses shows that

163 Alphaproteobacteria originated $2012 \mathrm{Ma}$ (95\% HPD 2284-1750 Ma) and $1786 \mathrm{Ma}$

164 (95\% HPD 2004-1574 Ma), based on the mito- and nuclear-encoded datasets,

165 respectively (Fig. S7), and diversified soon thereafter.

166 The topology of Alphaproteobacteria is another much-debated issue (Fan et al.,

167 2020; Luo, 2015; Muñoz-Gómez et al., 2019; Wang and Wu, 2015). We repeated the 
168 MCMCTree analysis by fixing the species phylogeny to 11 alternative topologies (Fig.

169 S1B). Most alphaproteobacterial orders showed highly consistent estimated ages (Fig.

170 2B). However, the posterior mean ages of Holosporales varied from 1800 to 1200

171 Ma across different topologies. This was because the alternative phylogenetic position

172 of Holosporales was a sister to Rickettsiales, in contrast to the topology used in the

173 main analysis where Holosporales branched within the Rhodospirillales (Fig. S1B).

174 Likewise, the origin time of Pelagibacterales showed considerable variations

175 depending on whether it formed a monophyletic group with the Rickettsiales. On the

176 other side, the impact of the topology of the eukaryotic tree (mitochondria subtree)

177 was relatively minor, as different topologies showed similar estimates of

178 alphaproteobacterial lineages at the order level (Fig. S8).

179 Traditionally, constructing the evolutionary timeline for bacteria is based on

180 fossils of cyanobacteria since bacterial fossils that can be accurately assigned to a

181 taxonomic group are only available for cyanobacteria (Schirrmeister et al., 2016).

182 Based on a best-selected scheme, the dates estimated using calibrations within

183 cyanobacteria were generally comparable to using the mitochondria-based strategy

184 (Fig. S9). However, with a slope of 0.251 in the infinite-sites plot (Fig. 2C), the

185 cyanobacteria-based strategy exhibited less precision compared with the

186 nuclear-encoded dataset (slope of 0.214 in the infinite-sites plot in Fig. 1). Further, 
187 analyses with different calibration schemes within cyanobacteria revealed larger

188 variations in the estimated ages for some clades (Fig. S9). For example, by using

189 different calibration schemes, we observed a spread of $444 \mathrm{Ma}$ for the posterior mean

190 age of the Rickettsiales crown group with the cyanobacteria-based strategy, in contrast

191 to 103 and $82 \mathrm{Ma}$ as estimated by the mito-encoded and nuclear-encoded datasets of

192 the mitochondria-based method, respectively (Fig. S9), likely underlined by the

193 abundant high-confidence fossils in eukaryotes and the shorter phylogenetic distance

194 between the mitochondrial lineage and Alphaproteobacteria.

195 The abundant eukaryotic fossils in our mitochondria-based strategy greatly

196 reduced dating uncertainty compared with the cyanobacteria-based approach. By

197 co-estimating the divergence times of both bacteria and their eukaryotic hosts, it

198 allows directly analyzing their co-evolution with the same dataset on the same tree,

199 thereby avoiding methodological artefacts stemming from comparing age estimates

200 from different studies. In addition, the mitochondria-based approach to date evolution

201 is conceptually distinct from the HGT-based method (Davín et al., 2018; Wolfe and

202 Fournier, 2018), as mitochondrial endosymbiosis is far more complex than simply an

203 HGT event (Roger et al., 2017; Sagan, 1967). Consequently, thanks to the many

204 high-confidence orthologs shared by mitochondrial and alphaproteobacterial lineages,

205 our taxon- and gene-rich multigene data mitigate the challenges faced by others (Shih 
et al., 2017) (see Supplementary Note S3.1). Nevertheless, our tentative analysis is

207

208

209

210

211

212

213

214

215

216

217

220

221

222

223

224

subject to other challenges such as the lack of internal fossils within the

Alphaproteobacteria and violation to molecular clock caused by the fast-evolving

nature of mitochondrial genomes. Although its impact should be attenuated by the

relaxed molecular clock model, it is important to keep this limit in mind when

interpreting the results. Further, the mitochondria-based strategy works well for

Alphaproteobacteria, but calibrating the evolution of related bacteria like Beta- and

Gammaproteobacteria with this strategy requires additional testing and

benchmarking.

The divergence times estimated here agree with some previous studies

(Battistuzzi et al., 2004; Battistuzzi and Hedges, 2009), but are much older than in

other studies that constrained the origin time of symbiotic or pathogenic bacteria to be

the same as that of their dominant modern hosts, thus ignoring the possibility of host

shifts (Chriki-Adeeb and Chriki, 2016; Luo et al., 2013; Weinert et al., 2009)

(Supplementary Note S3.2). Our results suggest that the origin of Rickettsiales, where

most extant members adapt to an animal-associated lifestyle, predated animals'

emergence for $\sim 700$ Ma but coincided with the origin of eukaryotes (Fig. 1).

Moreover, ancestral lifestyle reconstruction using BayesTraits (Meade and Pagel, 2016) with a much broader taxon sampling of Rickettsiales suggests the LCA of 
225 Rickettsiales to be symbionts of protists and that the transition rate from

226 protist-associated to animal-associated lifestyle was more than ten times higher than

227 that of the reverse process (Fig. 2D-E). This strongly challenges the view of the

228 concurrence of Rickettsiales and animals (Weinert et al., 2009), and instead suggests

229 frequent host transitions from unicellular eukaryotes to animals during Rickettsiales

230 evolution. Presumably, the predatory nature of early eukaryotes like amoebae or

231 ciliates might have helped forming primitive association with ancestral Rickettsiales

232 (Vannini et al., 2005). Later, animals could have acquired Rickettsiales parasites early

233 in their evolution by filter-feeding on infected protists (Supplementary Note S3.3).

234 This scenario is also supported by recent findings of the association between

235 Rickettsiales members with diverse protists (Castelli et al., 2016; Montagna et al.,

2362013 ) and by the presence of amoebae-derived genes in some Rickettsiales genomes

237 (Ogata et al., 2006). Holosporales are mostly intracellular parasites of the ciliate

238 Paramecium, but are increasingly found associated with a diverse range of protists

239 including amoebae, rhizarians and Euglenozoa (Muñoz-Gómez et al., 2019),

240 consistent with their deep divergence. Overall, our results are not contradictory to the

241 idea of host-bacteria co-evolution, but suggest more frequent host transitions than

242 previously understood and that caution should be exercised when assigning the

243 divergence time of bacteria based on that of their modern hosts. Besides, the ubiquity 
244 of microbial eukaryotes like amoebae in modern environments reminds people to pay

245 more attention to them as potential reservoirs of emerging human diseases.

\section{Methods}

\section{Phylogenomic reconstruction}

249 We followed Martijn et al., 2018, and used the 24 conserved genes encoded by both

250 the mitochondrial and alphaproteobacterial genomes annotated by MitoCOGs

251 (Kannan et al., 2014) to determine their phylogenetic relationship. Eighty genomes

252 were carefully selected for phylogenomic reconstruction based on prior studies

253 (Supplementary Note S1.1; Table S1). Genes were aligned using MAFFT v7.222

254 (Katoh and Standley, 2013) and trimmed with TrimAl v1.4 (“-st 0.001”)

255 (Capella-Gutiérrez et al., 2009). Because Alphaproteobacteria is subjected to strong

256 compositional heterogeneity across lineages (Luo, 2015; Martijn et al., 2018;

257 Muñoz-Gómez et al., 2019), which might confound phylogenetic signals and cause

258 phylogenetically unrelated species with similar GC content to cluster together, we

259 recoded the 20 amino acids into four nucleic acid characters according to their

260 physicochemical properties with the dayhoff4 and SR4 recoding scheme, respectively

261 (Luo, 2015; Martijn et al., 2018; Muñoz-Gómez et al., 2019). Phylogenomic

262 reconstruction was performed under the empirical profile mixture model 
264 ultrafast bootstraps using IQ-Tree v1.6.11 (Nguyen et al., 2015). As the trees obtained

265 by dayhoff4 and SR4 recoding schemes showed similar topologies (Fig. S1), we used

266 the one obtained by the dayhoff4 recoding scheme for dating (and we subsequently

267 included more eukaryotic taxa as mitochondrial lineages based on the general

268 consensus understanding of the eukaryotic phylogeny for both the mito- and

269 nuclear-encoded datasets in dating [see Fig. S2A and Supplementary Note S1.1]).

\section{Calibration information}

272 Four and ten calibration points within the eukaryote clade were selected for the mito-

273 and nuclear-encoded datasets, respectively (Fig. S2). We based the lower limit of a

274 calibration point upon the most ancient uncontroversial fossil from within the clade.

275 Since fossil records only tell the time that the group of interest had already appeared,

276 the actual origin time of a clade could be more ancient than these minima. Maximum

277 time constraints were determined from the youngest geological formation or

278 stratigraphic range without any members of the clade of interest, as used and

279 recommended by many studies (Betts et al., 2018; Donoghue and Benton, 2007; Dos

280 Reis et al., 2015). Several alternative time constraints were also considered to

281 accommodate the uncertainties in calibration. The full details of calibrations are given 


\section{2 in Supplementary Note S2.}

\section{Divergence time estimation}

285 We compiled two datasets for the mitochondria-based divergence time estimation of

286 Alphaproteobacteria, the mito-encoded dataset and the nuclear-encoded dataset. The

287 mito-encoded set was based on the aforementioned 24 orthologs conserved in

Alphaproteobacteria and mitochondrial lineages. For the nuclear-encoded set, we

retrieved the 29 genes that are likely transferred from the mitochondrial genome to the

nuclear genome during the early evolution of eukaryotes from Wang and $\mathrm{Wu}, 2015$,

291 and excluded seven genes involving putative paralogs (Dataset S1). Wrongly

292 annotated sequences in each alignment were removed by manually checking the

293 alignment and gene phylogeny.

294 To alleviate the impacts of mutational saturation, we used amino acids in the

295 main analysis but also repeated the analysis with nucleotide sequences (only the first

296 two codon positions). Dating analyses were predominantly carried out with the

297 approximate likelihood calculation with MCMCTree 4.9j (Yang, 2007), and we also

298 examined the consistency of the results with PhyloBayes v4.1b (Lartillot et al., 2009).

299 A constraint tree constructed using the dayhoff4 recoding scheme described above 
301 Because previous studies often came to different topologies of the

302 Alphaproteobacteria phylogeny, which are mainly associated with the positions of

303 mitochondria, Holosporales and Pelagibacterales (Fan et al., 2020; Luo, 2015;

304 Martijn et al., 2018; Muñoz-Gómez et al., 2019), we considered two, two, and three

305 distinct topologies for these three orders respectively, totaling $2 \times 2 \times 3=12$

306 topologies (Fig. S1B; Supplementary Note S1.2). We addressed the topology

307 uncertainty by repeating MCMCTree analysis with each alternative topology. We

308 further selected a best-practiced dating scheme by investigating the impact of the

309 calibration information, clock model, number of partitions, and cross-lineage rate

310 variation on the estimated posterior ages (see Supplementary Note S1.2 for details).

311 The burn-in, sampling frequency, and number of the iterations were adjusted to

$312100,000,100$, and 20,000, respectively, based on the results of testing runs. This

313 ensured that the effective sample size for all parameters were above 200, as

314 commonly recommended for MCMC-based Bayesian phylogenetic inference

315 (Nascimento et al., 2017). Convergence was assessed by comparing the posterior

316 means from two independent chains and with Tracer v1.6

317 (http://tree.bio.ed.ac.uk/software/tracer/). The posterior ages were compared with

318 effective priors ("usedata $=0$ ") to ensure that their distributions were different and

319 thereby the sequences used in MCMCTree analysis were informative (Table S2). 
320 Further, we followed the above procedure to date the divergence time of

321 Alphaproteobacteria using the traditional strategy where all calibration points were

322 placed within cyanobacteria for comparison (see Supplementary Note S2.2).

323

\section{Data availability}

325 All of the sequences, phylogenetic trees, molecular dating analysis results, and the

326 Ruby (Goto et al., 2010) codes generating them, are available at

327 https://figshare.com/s/ad430d1f5bace9eb2000.

329 Acknowledgements

330 We are particularly grateful to Jan Janouškovec from University of Oslo for his

331 insights on endosymbiosis and constructive comments on the draft of the manuscript.

332 We thank Mario dos Reis from QMUL, Richard Brown from LJMU, Charles Foster

333 from USYD, and Joana Wolfe from MIT for guidance in molecular dating, and Sergio

334 Muñoz-Gómez from Dalhousie University for help in phylogenomics analysis. We

335 thank lab members Tianhua Liao and Hao Zhang for discussion, and Kwok Chu

336 Cheung for data retrieving.

337 This work is supported by the National Key R\&D Program of China

338 (2018YFC0309800), the Hong Kong Research Grants Council Area of Excellence 
339 Scheme (AoE/M-403/16), the Direct Grant of CUHK (4053257 \& 3132809) and The

340 CUHK Impact Scheme Fellowship to (S.W.).

\section{References}

343 Battistuzzi FU, Feijao A, Hedges SB. 2004. A genomic timescale of prokaryote

344 evolution: Insights into the origin of methanogenesis, phototrophy, and the

$345 \quad$ colonization of land. BMC Evol Biol 4:44. doi:10.1186/1471-2148-4-44

346 Battistuzzi FU, Hedges SB. 2009. A major clade of prokaryotes with ancient

347 adaptations to life on land. Mol Biol Evol 26:335-343.

348 doi:10.1093/molbev/msn247

349 Betts HC, Puttick MN, Clark JW, Williams TA, Donoghue PCJ, Pisani D. 2018.

350 Integrated genomic and fossil evidence illuminates life's early evolution and 351 eukaryote origin. Nat Ecol Evol 2:1556-1562. doi:10.1038/s41559-018-0644-х

352 Boussau B, Karlberg EO, Frank AC, Legault BA, Andersson SGE. 2004.

353 Computational inference of scenarios for alpha-proteobacterial genome evolution.

$354 \quad$ Proc Natl Acad Sci U S A 101:9722-9727. doi:10.1073/pnas.0400975101

355 Butterfield NJ. 2015. Early evolution of the Eukaryota. Palaeontology.

356 doi:10.1111/pala.12139

357 Capella-Gutiérrez S, Silla-Martínez JM, Gabaldón T. 2009. trimAl: A tool for 
automated alignment trimming in large-scale phylogenetic analyses.

Castelli M, Sassera D, Petroni G. 2016. Biodiversity of “non-model” Rickettsiales and their association with aquatic organismsRickettsiales: Biology, Molecular doi:10.1007/978-3-319-46859-4_3

Chriki-Adeeb R, Chriki A. 2016. Estimating divergence times and substitution rates in Rhizobia. Evol Bioinforma 12:87-97. doi:10.4137/EBO.S39070

Davín AA, Tannier E, Williams TA, Boussau B, Daubin V, Szöllosi GJ. 2018. Gene doi:10.1038/s41559-018-0525-3

Donoghue PCJ, Benton MJ. 2007. Rocks and clocks: calibrating the Tree of Life using fossils and molecules. Trends Ecol Evol. doi:10.1016/j.tree.2007.05.005 2015. Uncertainty in the Timing of Origin of Animals and the Limits of Precision in Molecular Timescales. Curr Biol 25:2939-2950.

375 Drummond AJ, Ho SYW, Phillips MJ, Rambaut A. 2006. Relaxed phylogenetics and 376 dating with confidence. PLoS Biol 4:699-710. doi:10.1371/journal.pbio.0040088 
377 Eme L, Sharpe SC, Brown MW, Roger AJ. 2014. On the Age of Eukaryotes:

378 Evaluating Evidence from Fossils and Molecular Clocks. Cold Spring Harb

380 Ettema TJG, Andersson SGE. 2009. The $\alpha$-proteobacteria: The Darwin finches of the

381 bacterial world. Biol Lett 5:429-432. doi:10.1098/rsbl.2008.0793

382 Fan L, Wu D, Goremykin V, Xiao J, Xu Y, Garg S, Zhang C, Martin WF, Zhu R.

383 2020. Phylogenetic analyses with systematic taxon sampling show that

doi:10.1038/s41559-020-1239-x

386

387

388

390

391

392

393

394

395 mitochondria branch within Alphaproteobacteria. Nat Ecol Evol 4:1213-1219.

Fernandes NM, Schrago CG. 2019. A multigene timescale and diversification dynamics of Ciliophora evolution. Mol Phylogenet Evol 139:106521. doi:10.1016/j.ympev.2019.106521

Goto N, Prins P, Nakao M, Bonnal R, Aerts J, Katayama T. 2010. BioRuby: Bioinformatics software for the Ruby programming language. Bioinformatics 26:2617-2619. doi:10.1093/bioinformatics/btq475

Hedges SB, Blair JE, Venturi ML, Shoe JL. 2004. A molecular timescale of eukaryote evolution and the rise of complex multicellular life. BMC Evol Biol 4:2. doi:10.1186/1471-2148-4-2

Kannan S, Rogozin IB, Koonin E V. 2014. MitoCOGs: Clusters of orthologous genes 
Biol 14:237. doi:10.1186/s12862-014-0237-5

Katoh K, Standley DM. 2013. MAFFT Multiple Sequence Alignment Software 30:772-780. 25:2286-2288. doi:10.1093/bioinformatics/btp368

404

Luo H. 2015. Evolutionary origin of a streamlined marine bacterioplankton lineage. ISME J 9:1423-1433. doi:10.1038/ismej.2014.227 strategies in marine alphaproteobacteria. MBio 4:e00373-13.

408 doi:10.1128/mBio.00373-13

409

Martijn J, Vosseberg J, Guy L, Offre P, Ettema TJG. 2018. Deep mitochondrial origin outside the sampled alphaproteobacteria. Nature 557:101-105. doi:10.1038/s41586-018-0059-5

412 Meade A, Pagel M. 2016. BayesTraits V3 Manual.

413 Merhej V, Raoult D. 2011. Rickettsial evolution in the light of comparative genomics. 
415 Montagna M, Sassera D, Epis S, Bazzocchi C, Vannini C, Lo N, Sacchi L, Fukatsu T,

416 Petroni G, Bandi C. 2013. "Candidatus Midichloriaceae” fam. Nov.

417 (Rickettsiales), an ecologically: Widespread clade of intracellular

$418 \quad$ alphaproteobacteria. Appl Environ Microbiol 79:3241-3248.

419 doi:10.1128/AEM.03971-12

420

421

422

423

424 Nascimento FF, dos Reis M, Yang ZH. 2017. A biologist's guide to Bayesian

425

426

427

428

429

430

431

432

433

Muñoz-Gómez SA, Hess S, Burger G, Franz Lang B, Susko E, Slamovits CH, Roger AJ. 2019. An updated phylogeny of the alphaproteobacteria reveals that the parasitic rickettsiales and holosporales have independent origins. Elife 8:e42535. doi:10.7554/eLife.42535

phylogenetic analysis. Nat Ecol Evol 1:1446-1454.

doi:10.1038/s41559-017-0280-x

Nei M, Xu P, Glazko G. 2001. Estimation of divergence times from multiprotein sequences for a few mammalian species and several distantly related organisms. Proc Natl Acad Sci U S A 98:2497-2502. doi:10.1073/pnas.051611498

Nguyen LT, Schmidt HA, Von Haeseler A, Minh BQ. 2015. IQ-TREE: A fast and effective stochastic algorithm for estimating maximum-likelihood phylogenies. Mol Biol Evol 32:268-274. doi:10.1093/molbev/msu300

Ogata H, La Scola B, Audic S, Renesto P, Blanc G, Robert C, Fournier PE, Claverie 
$434 \quad$ JM, Raoult D. 2006. Genome sequence of Rickettsia bellii illuminates the role of

435 amoebae in gene exchanges between intracellular pathogens. PLoS Genet

$436 \quad 2: 733-744$. doi:10.1371/journal.pgen.0020076

437 Parfrey LW, Lahr DJG, Knoll AH, Katz LA. 2011. Estimating the timing of early

438 eukaryotic diversification with multigene molecular clocks. Proc Natl Acad Sci

439 U S A 108:13624-13629. doi:10.1073/pnas.1110633108

440 Rannala B, Yang Z. 2007. Inferring speciation tunes under an episodic molecular

$441 \quad$ clock. Syst Biol 56:453-466. doi:10.1080/10635150701420643

442 Rodríguez-Ezpeleta N, Embley TM. 2012. The SAR11 group of alpha-proteobacteria

443 is not related to the origin of mitochondria. PLoS One 7:e30520.

$444 \quad$ doi:10.1371/journal.pone.0030520

445 Roger AJ, Muñoz-Gómez SA, Kamikawa R. 2017. The Origin and Diversification of

446 Mitochondria. Curr Biol. doi:10.1016/j.cub.2017.09.015

447 Sagan L. 1967. On the origin of mitosing cells. J Theor Biol 14:225-274.

448 doi:10.1016/0022-5193(67)90079-3

449 Schirrmeister BE, Sanchez-Baracaldo P, Wacey D. 2016. Cyanobacterial evolution

450 during the Precambrian. Int J Astrobiol 15:187-204.

$451 \quad$ doi:10.1017/S1473550415000579

452 Schrallhammer M, Castelli M, Petroni G. 2018. Phylogenetic relationships among 
453 endosymbiotic R-body producer: Bacteria providing their host the killer trait.

$454 \quad$ Syst Appl Microbiol 41:213-220. doi:10.1016/j.syapm.2018.01.005

455 Shih PM, Hemp J, Ward LM, Matzke NJ, Fischer WW. 2017. Crown group

456 Oxyphotobacteria postdate the rise of oxygen. Geobiology 15:19-29.

457 doi:10.1111/gbi.12200

458 Sun Y, Powell KE, Sung W, Lynch M, Moran MA, Luo HW. 2017. Spontaneous

459 mutations of a model heterotrophic marine bacterium. Isme J 11:1713-1718.

460 doi:10.1038/ismej.2017.20

461 Vannini C, Petroni G, Verni F, Rosati G. 2005. A bacterium belonging to the

462 Rickettsiaceae family inhabits the cytoplasm of the marine ciliate Diophrys

463 appendiculata (Ciliophora, Hypotrichia). Microb Ecol 49:434-442.

464 doi:10.1007/s00248-004-0055-1

465 Wang S, Meade A, Lam H-M, Luo H. 2020. Evolutionary Timeline and Genomic

466 Plasticity Underlying the Lifestyle Diversity in Rhizobiales. mSystems

$467 \quad$ 5:e00438-20. doi:10.1128/mSystems.00438-20

468 Wang Z, Wu M. 2015. An integrated phylogenomic approach toward pinpointing the

469 origin of mitochondria. Sci Rep 5:7949. doi:10.1038/srep07949

470 Weinert LA, Werren JH, Aebi A, Stone GN, Jiggins FM. 2009. Evolution and

471 diversity of Rickettsia bacteria. BMC Biol 7:6. doi:10.1186/1741-7007-7-6 
472 Wolfe JM, Fournier GP. 2018. Horizontal gene transfer constrains the timing of

473 methanogen evolution. Nat Ecol Evol 2:897-903.

474 doi:10.1038/s41559-018-0513-7

475 Yang D, Oyaizu Y, Oyaizu H, Olsen GJ, Woese CR. 1985. Mitochondrial origins.

$476 \quad$ Proc Natl Acad Sci U S A 82:4443-4447. doi:10.1073/pnas.82.13.4443

477 Yang Z. 2007. PAML 4: Phylogenetic analysis by maximum likelihood. Mol Biol

$478 \quad$ Evol 24:1586-1591. doi:10.1093/molbev/msm088

479

480 


\section{$481 \quad$ Figure legends}

482

485

487

492

496

497

\section{Figure 1. Divergence time estimate using the mitochondria-based strategy}

The evolutionary timeline of the Alphaproteobacteria estimated using the

best-practiced dating scheme for the mito-encoded (the top) and nuclear-encoded (the bottom) datasets. Node bars denote the 95\% HPD interval of estimated dates. Nodes

with red circles denote calibration points. The cartoon graphs above the timeline indicate the origin times of eukaryotic lineages that Rickettsiales and Holosporales

are mostly associated with, inferred simultaneously with Alphaproteobacteria using the same dataset. The potential time range of mitochondrial endosymbiosis is also

indicated. The panels in the upper and lower left corners are the infinite-sites plots, where the uncertainty in the divergence time (measured as the 95\% HPD width) is plotted against the posterior mean of estimated times for each node. A lower value of the slope indicates less changes in the 95\% HPD width, hence higher precision in dating. The sources and credits of the cartoon graphs are provided in the online open access repository FigShare (see Data availability).

Figure 2. Comparison of the estimated times with alternative dating strategies using MCMCTree and ancestral lifestyle reconstruction of Rickettsiales. (A) The divergence times estimated using alternative schemes (y-axis; see Dataset S2) versus using the 
500 best-practiced scheme (x-axis). The best-practiced scheme used a full partition and

501 autocorrelated rates clock model (Dataset S2). The bars in blue indicate the $95 \%$

502 HPDs. Phan: only calibration points with Phanerozoic fossils considered; Max-1:

503 maximum constraints for nodes with controversial maximum ages removed; Root-1: a

504 more conservative root age; Single partition: all sequences considered as a single

505 partition; IR: independent rates clock model (Dataset S2). (B) Changes in the

506 estimated times (95\% HPD) that result from using different species tree topologies

507 (Fig. S1C). The detailed posterior dates for each clade are shown in Table S2. (C) The

508 infinite-sites plot using the cyanobacteria-based strategy. (D) Inferred ancestral hosts

509 of Rickettsiales. The pie charts on the nodes show the estimated probabilities of the

510 hosts, and the branch colors indicate the hosts with the higher probability at the

511 corresponding node. Tips represent the randomly selected representative of each OTU

512 (defined by $97 \%$ identity of $16 \mathrm{~S}$ rRNA gene). (E) The transition rates from

513 animal-associated to protist-associated $\left(\mathrm{q}_{\mathrm{AP}}\right)$ and from protist-associated to

514 animal-associated ( $\left.\mathrm{q}_{\mathrm{PA}}\right)$ estimated by the MCMC method in BayesTraits multistate.

515 The $\log$-transformed Bayes factor $(\log B F)$ is indicated, where values above 10 are

516 considered very strong evidence for support (Meade and Pagel, 2016). The sources

517 and credits of the cartoon graphs are provided in the online open access repository

518 FigShare (see Data availability). 


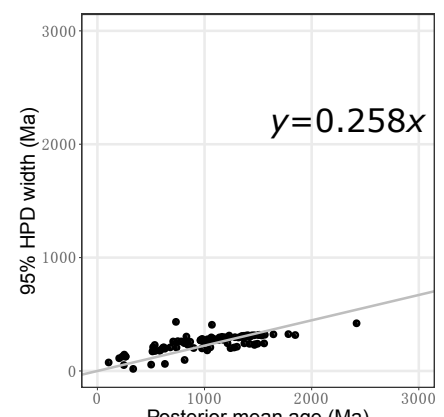

\section{mito-encoded}

\section{nuclear-encoded}

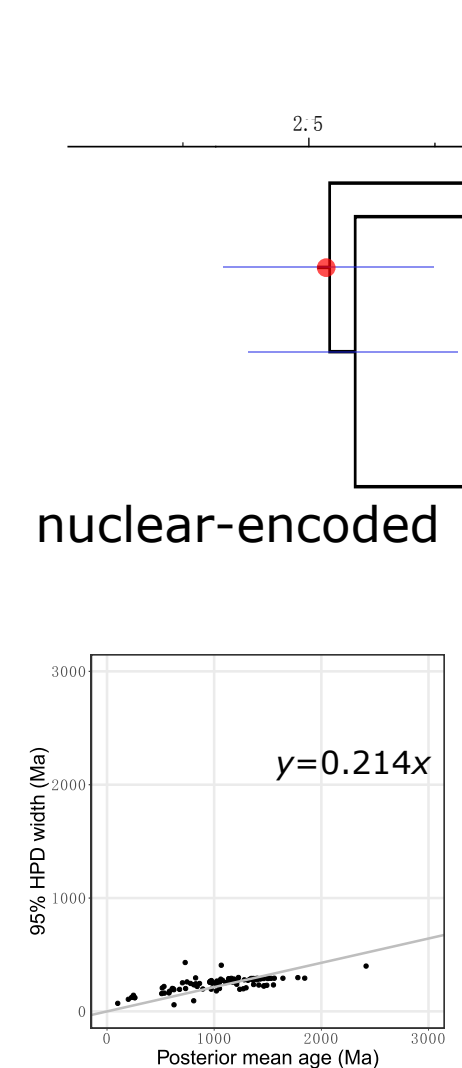

Posterior mean age (Ma)

Posterior mean age (Ma)

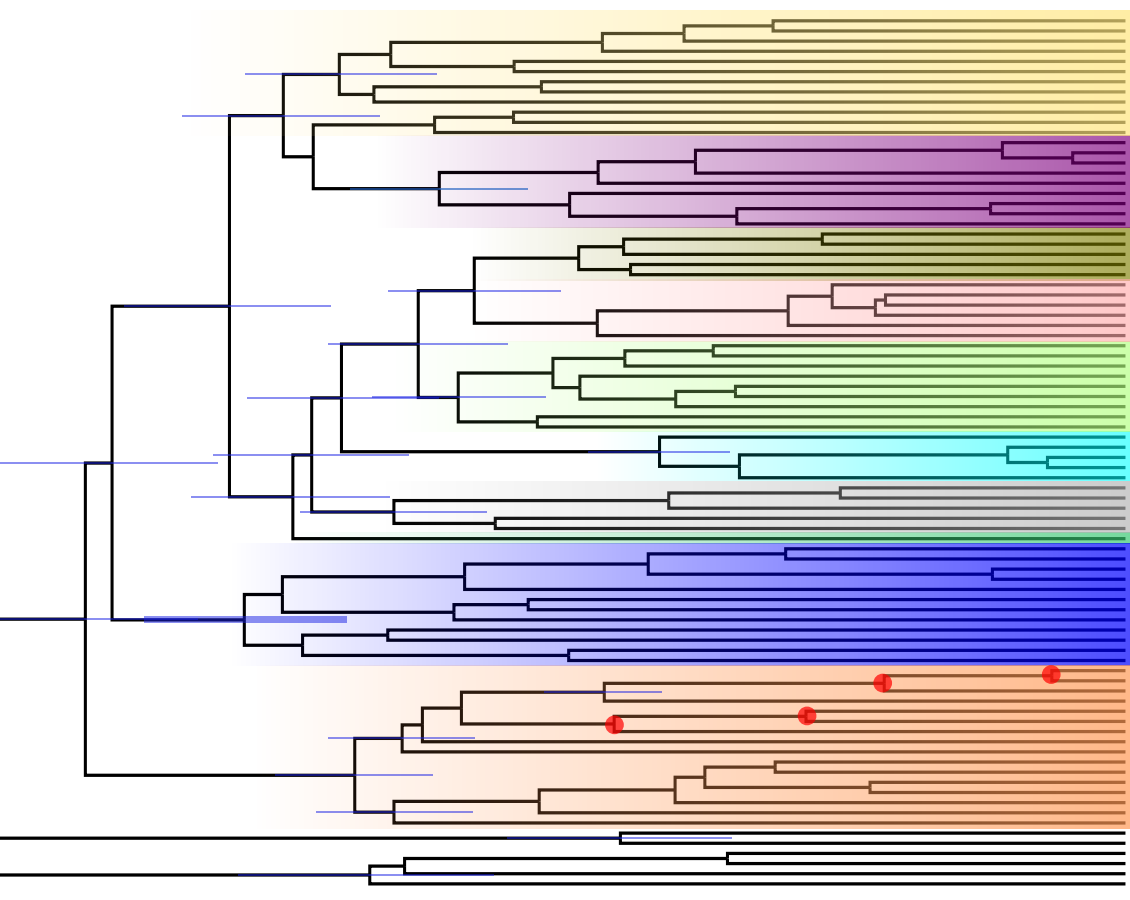

Paleoproterozoic

LCA of eukaryotes Amoebae

Rhodospirillales

Holosporales

Caulobacterales

Rhodobacterales

Rhizobiales

Pelagibacterales

Sphingomonadales

Sneathiellales

Anaplasmatacea

Midichloriaceae

Archaeplastida

Mitochondria

Jakobids

outgroup

Phanerozoic

Vertebrates

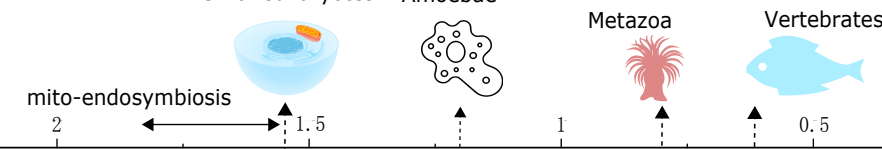

SAR

Archaeplastida

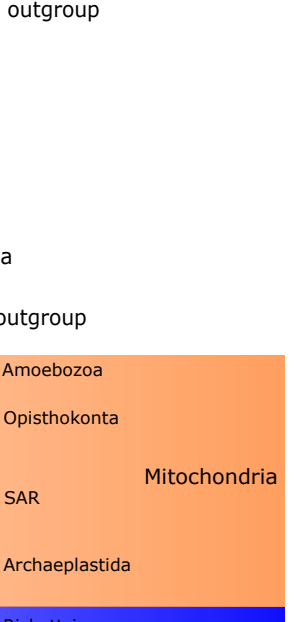

Rickettsiace

Anaplasmatace

Sneathiellales

Sphingomonadales

Pelagibacterales

Rhizobiales

Rhodobacterales

Caulobacterales

Holosporales

Rhodospirillales 
A
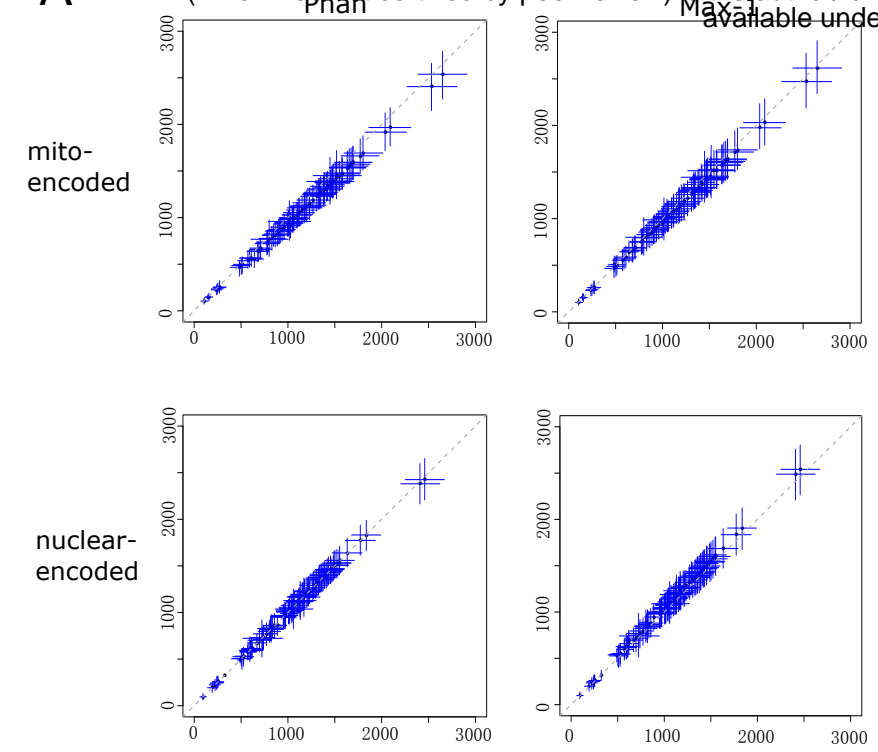
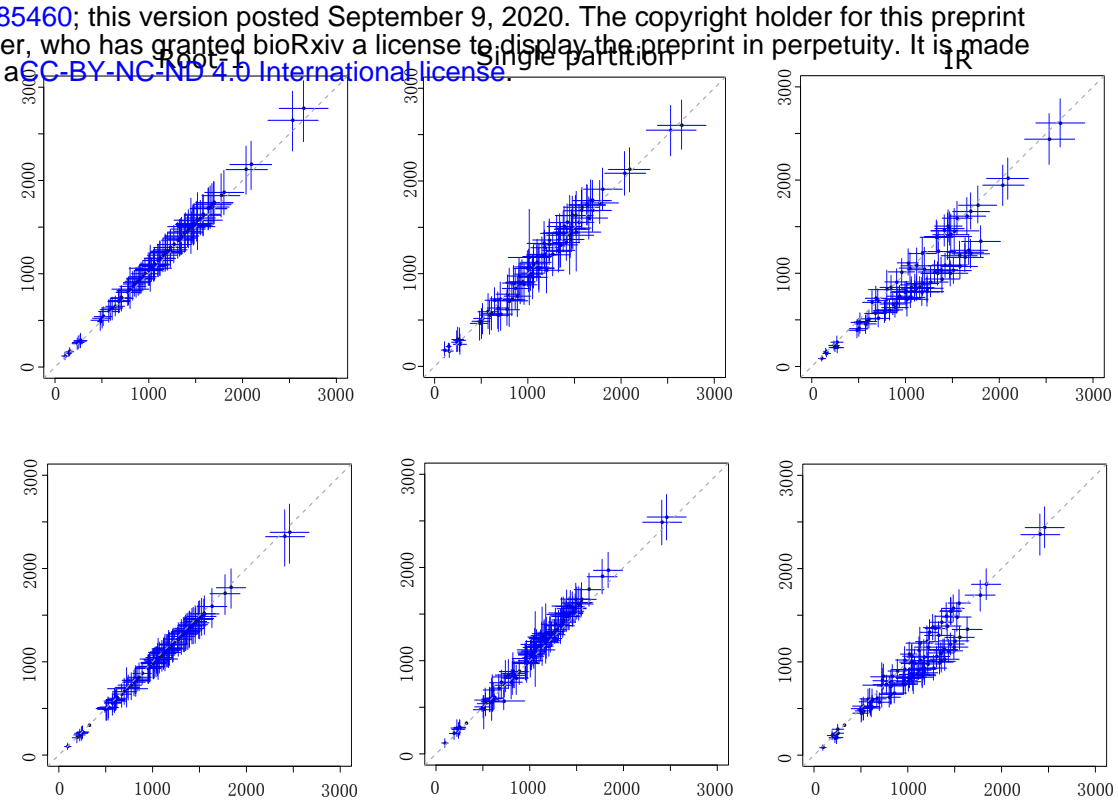

B

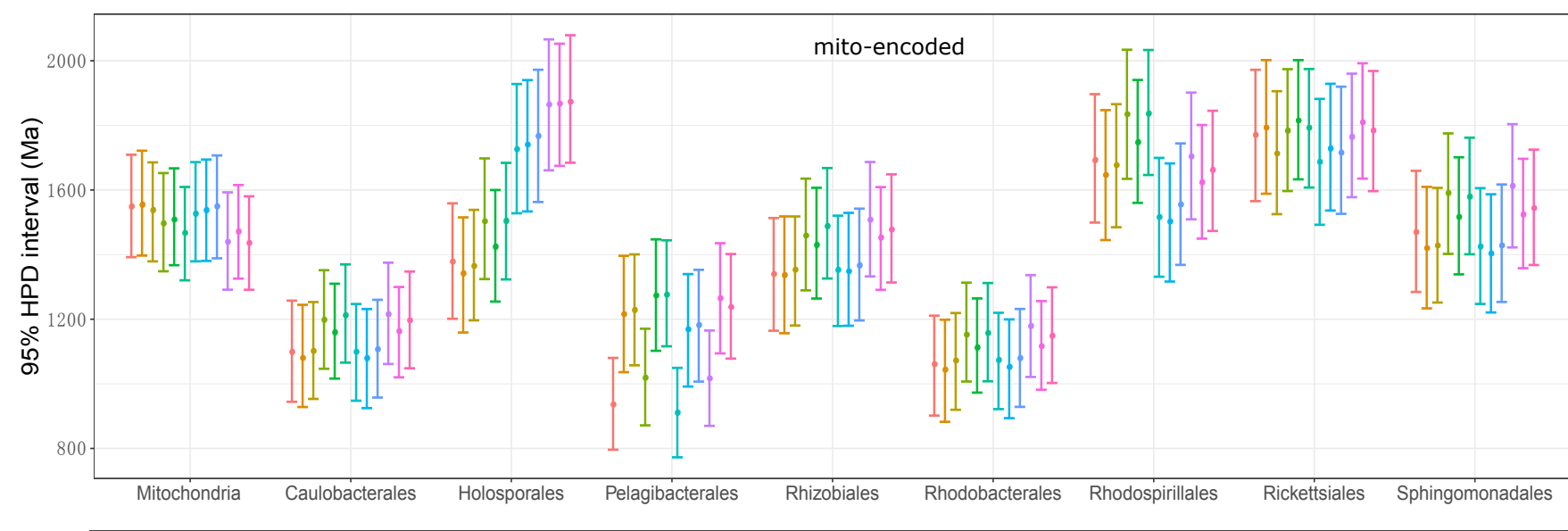

- Topo1

$\rightarrow$ Topo2

$\rightarrow$ Topo3

- Topo4

$\rightarrow$ Topo5

$\rightarrow$ Topo6

- Topo7

$\rightarrow$ Topo8

$\approx$ Topo10

$\Rightarrow$ Topo11

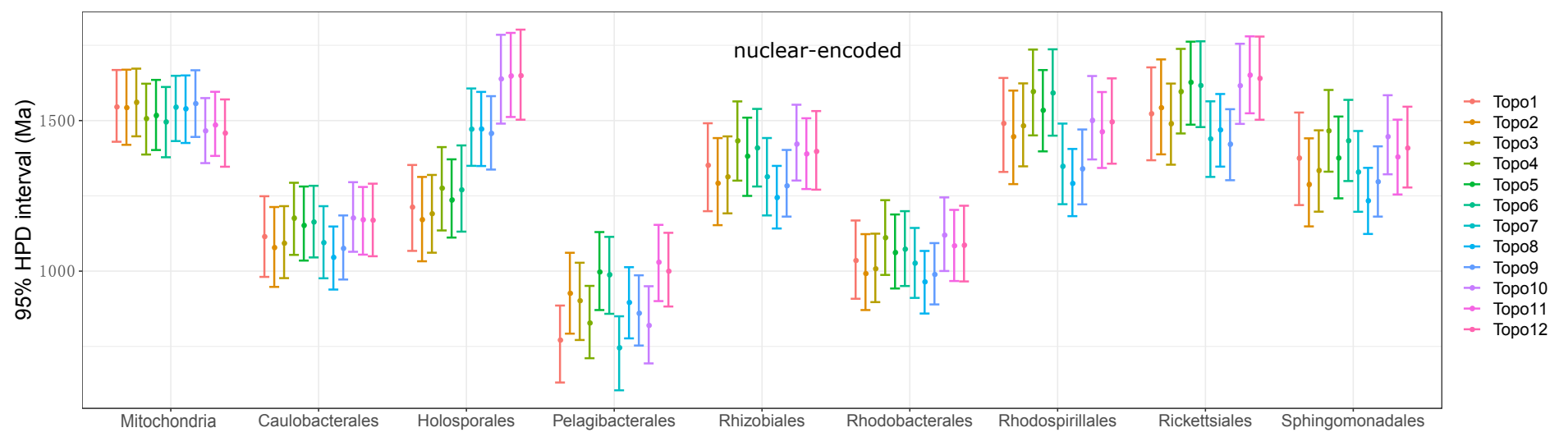

C

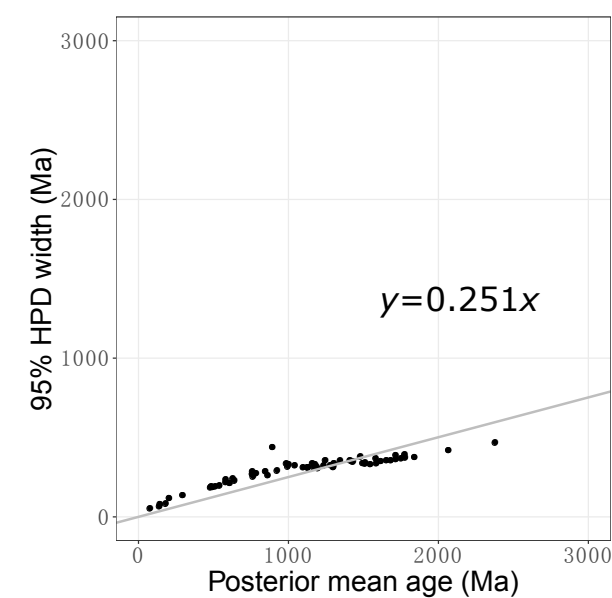

D protist-associated

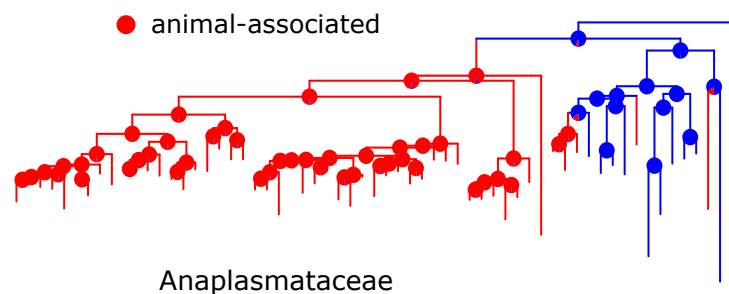

Midichloriaceae

E

animal-associated $\log B F=11.1$

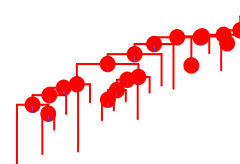

उक्षिक

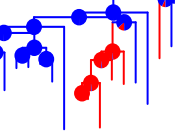

outgroup

Rickettsiaceae

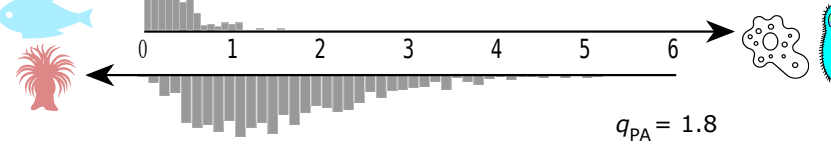

